\title{
Recent mobility of plastid encoded group II introns and twintrons in five strains of the unicellular red alga Porphyridium
}

Marie-Mathilde Perrineau, Dana Price, Georg Mohr, Debashish Bhattacharya

Group II introns are closely linked to eukaryote evolution because nuclear spliceosomal introns and the small RNAs associated with the spliceosome are thought to trace their ancient origins to these mobile elements. Therefore, elucidating how group II introns move, and how they lose mobility can potentially shed light on fundamental aspects of eukaryote biology. To this end, we studied five strains of the unicellular red alga Porphyridium purpureum that surprisingly contain 42 group II introns in their plastid genomes. We focused on a subset of these introns that encode mobility-conferring intronencoded proteins (IEPs) and found them to be distributed among the strains in a lineagespecific manner. The reverse transcriptase and maturase domains were present in all lineages but the DNA endonuclease domain was deleted in vertically inherited introns, demonstrating a key step in the loss of mobility. P. purpureum plastid intron RNAs had a classic group IIB secondary structure despite variability in the DIII and DVI domains. We report for the first time the presence of twintrons (introns-within-introns, derived from the same mobile element) in Rhodophyta. The P. purpureum IEPs and their mobile introns provide a valuable model for the study of mobile retroelements in eukaryotes and offer promise for biotechnological applications. 
2 Recent mobility of plastid encoded group II introns and twintrons in

3 five strains of the unicellular red alga Porphyridium

7 Marie-Mathilde Perrineau ${ }^{1}$, Dana C. Price ${ }^{1}$, Georg Mohr ${ }^{2}$, Debashish Bhattacharya ${ }^{1,3^{*}}$

$9{ }^{1}$ Department of Ecology, Evolution and Natural Resources, Rutgers University, New Brunswick,

10 New Jersey, 08901, USA

$11{ }^{2}$ Institute for Cellular and Molecular Biology, University of Texas at Austin, Austin, TX 78712 ,

12 USA

$13{ }^{3}$ Department of Marine and Coastal Sciences, Rutgers University, New Brunswick, New Jersey, 14 08901, USA

$17{ }^{*}$ Corresponding author: Debashish Bhattacharya, Department of Ecology, Evolution and Natural

18 Resources, Rutgers University, 59 Dudley Road, 102 Foran Hall, New Brunswick, NJ 08901,

19 USA. Telephone: +1 848-932-6218;

20 Fax: +1 732-932-8746; E-mail: debash.bhattacharya@gmail.com 


\section{Abstract}

23 Group II introns are closely linked to eukaryote evolution because nuclear spliceosomal introns

24 and the small RNAs associated with the spliceosome are thought to trace their ancient origins to

25 these mobile elements. Therefore, elucidating how group II introns move, and how they lose

26 mobility can potentially shed light on fundamental aspects of eukaryote biology. To this end, we

27 studied five strains of the unicellular red alga Porphyridium purpureum that surprisingly contain

2842 group II introns in their plastid genomes. We focused on a subset of these introns that encode

29 mobility-conferring intron-encoded proteins (IEPs) and found them to be distributed among the

30 strains in a lineage-specific manner. The reverse transcriptase and maturase domains were

31 present in all lineages but the DNA endonuclease domain was deleted in vertically inherited

32 introns, demonstrating a key step in the loss of mobility. P. purpureum plastid intron RNAs had

33 a classic group IIB secondary structure despite variability in the DIII and DVI domains. We

34 report for the first time the presence of twintrons (introns-within-introns, derived from the same

35 mobile element) in Rhodophyta. The P. purpureum IEPs and their mobile introns provide a

36 valuable model for the study of mobile retroelements in eukaryotes and offer promise for

37 biotechnological applications. 


\section{Introduction}

40 Nuclear genome evolution and eukaryotic cell biology in general are closely tied to the origin

41 and spread of autocatalytic group II (GII) introns. These parasitic genetic elements are thought to

42 have initially entered the eukaryotic domain through primary mitochondrial endosymbiosis (e.g.,

43 Rogozin et al. 2012; Doolittle 2014), and are implicated as a selective force behind formation of

44 the nuclear compartment (Aravind, Iyer and Koonin 2006, Martin and Koonin 2006). Ultimately,

45 GII introns were transferred to the nucleus and gave birth to the forerunners of nuclear

46 spliceosomal introns and the small RNAs associated with the spliceosome (Cech, 1986; Sharp,

47 1991; Qu et al. 2014). This explanation of intron origin, although widely held to be true (e.g.,

48 Rogozin et al. 2012) is nonetheless shrouded in the mists of evolutionary time. Understanding

49 more recent cases of GII intron gain and loss are vital to testing ideas about the biology of

50 autocatalytic introns. Here we studied GII intron evolution in five closely related strains of the

51 unicellular red alga Porphyridium purpureum (Rhodophyta) that surprisingly contain over 40

52 intervening sequences in their plastid genomes (Tajima et al. 2014). Red algae are not only

53 interesting in their own account as a taxonomically rich group of primary producers (Ragan et al.

54 1994; Bhattacharya et al. 2013) but they also contributed their plastid to a myriad of chlorophyll

$55 c$-containing algae such as diatoms, haptophytes, and cryptophytes through secondary

56 endosymbiosis (Bhattacharya et al.; 2004; Archibald, 2009). Therefore, GII introns resident in

57 red algal plastid genomes could also have entered other algal lineages through endosymbiosis.

58 With these ideas in mind, we explored the genetic diversity, secondary structure, and

59 evolution of GII introns and their mobility-conferring intron-encoded proteins (IEPs; Lambowitz

60 and Zimmerly, 2011) in the plastid genome of five strains of $P$. purpureum, four of which were

61 determined for this study. Phylogenetic analyses show that the P. purpureum IEPs and their 
62 introns are monophyletic, suggesting a shared evolutionary history (Toro and Martínez-Abarca,

63 2013). Analysis of IEPs reveals key traits associated with GII intron mobility and loss, and

64 analysis of secondary structures uncover unique features of red algal group II introns. We also

65 report for the first time the presence of twintrons (introns-within-introns) in Rhodophyta plastid

66 genomes and deduce their recent origins from existing IEPs that targeted heterologous DNA sites.

67 In summary, our study identifies a promising red algal model for the study of GII intron biology

68 and evolution and suggests these mobile elements could potentially be harnessed for

69 biotechnological applications (Enyeart et al. 2014).

\section{Materials and Methods}

Porphyridium purpureum strains and plastid genomes

Four Porphyridium purpureum strains (SAG 1380-1a, SAG 1380-1b, SAG 1380-1d [obtained

from the Culture Collection of Algae, Göttingen University] and CCMP 1328 [from the National

75 Center for Marine Algae and Microbiota, East Boothbay, ME]) were grown under sterile conditions on Artificial Sea Water (Jones et al. 1963) at $25^{\circ} \mathrm{C}$, under continuous light $(100 \mu \mathrm{mol}$

77 photons $\cdot \mathrm{m}^{-2} \cdot \mathrm{s}^{-1}$ ) on a rotary shaker at $100 \mathrm{rpm}$ (Innova 43, New Brunswick Eppendorf, Enfield, CT). Cells were pelleted via centrifugation and DNA was extracted from ca. $100 \mathrm{mg}$ of material with the DNeasy Plant Mini Kit (Qiagen) following the manufacturer's protocol. Sequencing libraries were prepared for each strain using the Nextera DNA Sample Preparation Kit (Illumina

81 Inc, San Diego, CA) and sequenced on an Illumina MiSeq sequencer using a 300-cycle (150x150

82 paired-end) MiSeq Reagent Kit v2 (Illumina, Inc.). Sequencing reads were quality and adapter

83 trimmed ( $\mathrm{Q}$ limit cutoff $=0.05)$ and overlapping pairs were merged at 3' end overlap using the

84 CLC Genomics Workbench 6.5.1 (CLC Bio, Aarhus, Denmark). 


\section{Mapping, polymorphism detection and analysis}

87 The reads from each of the four newly-sequenced strains above were mapped to the existing P. purpureum plastid reference genome (strain NIES 2140; Tajima et al. 2014) with a stringency of $90 \%$ sequence identity over a $90 \%$ read length fraction using the CLC Genomics Workbench (CLC Bio, Aarhus, DK). SNPs were called using the Genomics Workbench 6.5.1 quality-based variant detection ( $\geq 10 \mathrm{x}$ base coverage, quality score $>30$ and $\geq 50 \%$ frequency required to be called). An uncorrected distance phylogeny was constructed using a matrix of DNA polymorphisms detected between the five plastid genomes with the program MEGA6.06

94 (Tamura et al. 2013; 100 bootstrap replicates).

\section{Group II intron and IEP identification}

97 Novel GII introns in the plastid genomes of the four P. purpureum strains were identified by 98 aligning de-novo assembled (using the CLC Genomics Workbench v.6.5.1 de-novo assembler)

99 plastid contigs from each strain to the NIES 2140 reference. Multiple large ( $>50 \mathrm{bp})$ insertions

100 were identified in our denovo contigs with respect to the reference, and were annotated as

101 putative introns. We then mapped the corresponding raw short read data to these contigs and 102 manually inspected the mapping for assembly artifacts. Intron encoded proteins (IEPs) were

103 identified within the putative introns by ORF detection using the bacterial/plastidic genetic code.

104 The four domains that constitute an IEP (i.e., reverse transcriptase [RT], maturase [X], DNA-

105 binding [D], and endonuclease [En] [Mohr et al. 1993; San Filippo and Lambowitz, 2000]) were

106 identified by sequence alignment using ClustalX (Larkin et al. 2007) to known IEPs of the

107 prokaryote CL1/CL2 group and to those from the Rhodophyta, Viridiplantae, Cryptophyta, 
108 Euglenozoa, and stramenopiles (listed in Table S1) obtained from NCBI and the Group II intron

109 database (Dai et al. 2003; http://webapps2.ucalgary.ca/ groupii/, accessed Sept. 2014). To

110 examine the phylogeny of these mobile elements, the IEP peptide sequences were aligned with

111 the RT-domain alignment of Toro and Martínez-Abarca (2013) and maximum likelihood

112 phylogenies were inferred under the WAG amino acid substitution model with 100 bootstrap

113 replicates using MEGA6.06. The GII intron/IEP sequences described here are accessible using

114 NCBI accession numbers KKJ826367 to KKJ826395 and the $P$. purpureum plastid genome

115 under NC_023133 (Tajima et al. 2014).

116

117 Intron structure and evolution

118 Intron secondary structures were predicted using sequence alignment, manual domain

119 identification, and automatic structure conformation in comparison with previously predicted

120 structures of group IIB introns using the Mfold web server (Zuker, 2003; Table S1). A detailed

121 secondary structure model was generated based on the rpoCl intron and matld IEP (Fig. 1). This

122 was then used as a guide to predict draft structures using PseudoViewer3 (Byun and Han, 2009)

123 for all other GII introns. A domain alignment was then performed against the GII intron

124 structures derived from the cryptophyte Rhodomonas salina (Maier et al. 1995; Khan et al. 2007)

125 using ClustalX2.1, and a maximum-likelihood phylogeny was generated using intronic

126 nucleotide sequence data under the GTR $+\mathrm{I}+\Gamma \square$ model with 100 bootstrap replicates using

127 MEGA6.06 (Tajima et al. 2014). Prior to this, the IEPs or IEP remnants were removed to avoid

128 potential long-branch attraction artifacts. Additionally, conserved motifs within the basal DI,

129 DIV, DV and DVI domains (Table S2) were used as a BLASTN (Altschul et al. 1990) query to 
130 the five aligned plastid genomes to identify additional group II intron structures present in all

131 strains (and thus not identified via length heterogeneity upon initial assessment).

132 The twintrons present in the P. purpureum plastid genome were aligned and compared to

133 the other introns to allow identification of the outer and inner introns, exon binding sites, to

134 describe their secondary structures, and potentially to understand their mode of origin.

135

\section{Results and Discussion}

137 Paired-end short read sequencing of P. purpureum strains SAG 1380-1a, SAG1380-1b, SAG

$138 \quad 1380-1 \mathrm{~d}$ and CCMP1328 generated 5.5M, 3.4M, 2.7M and 4.3M reads, respectively, after

139 trimming and quality control. These data covered between 98 to 100 percent of the NIES2140

140 plastid reference genome (information regarding read mapping and coverage of the reference can

141 be found in Table 1). A phylogenetic tree of the five studied P. purpureum strains inferred on

142 the basis of 332 single nucleotide polymorphisms (SNPs) present in their plastid genomes

143 demonstrates the monophyly of the four strains reported here (SAG 1380-1a/b/d, CCMP-1328)

144 with respect to strain NIES 2140 (Fig. 2A; Tajima et al. 2014). By examining length

145 heterogeneity within these plastid genome sequence alignments, we identified four novel GII

146 intron/IEP combinations (mat lf, $1 g, 1 h, 1 i$; Table 2, Fig. 2B) in addition to the five previously

147 reported by Tajima et al. (2014; matla, $1 b, 1 c, 1 d$, $1 e)$. These novel elements exhibited lineage-

148 specific distributions on the phylogeny, whereas those encoding matla, $b, c$ and $e$ were

149 recovered from all strains (Fig. 2B). Using conserved structural motifs (see Fig. S1 and Materials

$150 \&$ Methods) as the basis for a homology search within remaining intronic and intergenic $P$.

151 purpureum plastid sequence, we defined two additional GII introns (within int $m n t A$, int.a rpoB),

152 and an intergenic element with GII intron structure located between the $p s b N$ and $p s b T$ genes. 
153 Each of the three structures is present in all four strains, and contain remnant (or 'ghost') ORFs

154 that have lost their IEPs via sequence degeneration or excision. These structures were

155 subsequently included in our analyses.

156 We identified six new GII intron insertion sites in our $P$. purpureum strains encoding the

$157 m a t l f a, l f b, l f c, l g, l h, l i$ IEPs (see Table 2 ) in addition to the five sites previously described in

158 the NIES 2140 strain (encoding mat la, $1 b, 1 c, 1 d$, 1 ; Tajima et al. 2014; see Fig. 2). Among the

159 nine GII intron/IEP combinations present, only four occur at the same insertion site in all strains

160 (matla $1 b, 1 c$, and $l e$ ), whereas four are unique to individual strains (matld, $1 g, 1 \mathrm{~h}$, and $1 \mathrm{i}$ ).

161 The matlfa and mat lfb IEPs are identical at the nucleotide level and form twintrons (see below),

162 whereas mat lfc contains a single SNP.

163 A maximum-likelihood phylogeny was constructed using an alignment of the novel GII

164 introns described in this study, along with the 42 introns present in NIES 2140 (with IEP

165 sequences removed from the alignment; Fig. 3). This analysis demonstrates that twelve IEP/IEP-

166 remnant containing GII introns in P. purpureum form an exclusive monophyletic group (88\%

167 bootstrap support), whereas the matla- and mat $1 b$-encoding elements are sister taxa in a

168 distantly related and evolutionarily diverged clade. Despite partial nucleotide sequence

169 conservation (Fig. S1), the intergenic structure encoding matla could not be folded into a

170 functional group II intron structure (only domains DIV-DVI could be identified (Fig. S2), and we

171 were unable to identify any group II intron secondary structural homology within the matlb-

172 encoding intron (see Fig. S1 and the section below entitled, 'Group IIB intron secondary

173 structure'). These structures may then represent "group II-like introns" as defined by Toro and

174 Nisa-Martinez (2014) in that they lack canonical secondary structures and yet maintain a

175 maturase domain. In addition, the GII intron structures with remnant or ghost ORFs recovered in 
176 our analysis formed a monophyletic group with those that maintained functional IEPs. These

177 results are consistent with the evolutionary model widely accepted for group II introns (Toor et

178 al. 2001; Simon et al. 2009) that predicts co-evolution of IEPs and self-splicing RNAs, and

179 suggests that IEP-lacking (remnant) introns derive from introns that once contained a functional

180 mobility-conferring enzyme.

181

182 Intron-encoded proteins

183 Intron-encoded proteins present at the same insertion site are nearly identical among the strains

184 (98.9-100\% amino acid identity), except for the mat $1 b$ IEP in strain NIES 2140 which has an

185 apparent truncation of 27 amino acids due to a premature stop-codon. All nine IEPs contain two

186 fully conserved reverse transcriptase (RT) and maturase (X) domains (Fig. S3), whereas four of

187 the five elements present in all five $P$. purpureum strains (matla, $1 b, 1 c, 1 e$ ) are either truncated

188 or have completely lost the DNA-binding (D) and endonuclease (EN) domains responsible for

189 conferring mobility (Simon et al. 2009). These latter GII introns thus appear to have lost mobility,

190 and exhibit vertical inheritance. Additionally, matla and mat $1 b$ lack the YADD motif crucial for

191 reverse transcriptase activity at the active site (Fig. S3; Moran et al. 1995). The remaining five

192 GII introns encoding matld, matlf[a,b,c], matlg, matlh, matli are distributed in lineage-specific

193 patterns on the $P$. purpureum phylogeny (Fig. 2A) and likely remain mobile because they retain

194 all functional domains (Fig. S1). Thereforre, we show here for the first time examples of recent

195 intron mobility and putative stability; the latter being represented by plastid-encoded IEPs that

196 lack a functional endonuclease domain due to mutation and/or sequence degeneration.

197 Phylogenetic analysis using the IEP peptide alignment shows that seven of the nine $P$.

198 purpureum IEPs form a monophyletic clade that is sister to cryptophyte plastid IEPs, the 
199 cyanobacterial CL2B clade, and Euglenozoa plastids (Figs. 4, S4). The mat $1 a$ and mat $1 b$ IEPs,

200 derived from group II introns found to lack typical secondary structure, create a paraphyletic

201 assemblage within the cryptophytes (matla) or group outside of the CL2B clade (mat 1b). This

202 tree, in association with Fig. 3, illustrates the shared ancestry and subsequent co-evolution of

203 seven IEPs as well as their associated GII intron structures.

205 Group IIB intron secondary structure

206 Self-splicing group II introns are dependent on a conserved secondary and tertiary RNA structure.

207 These autocatalytic genetic elements are composed of six distinct double-helical domains (DI to

208 DVI) that radiate from a central wheel with each domain having a specific activity (Lambowitz

209 and Zimmerly, 2011). As illustrated by the rpoCl GII intron that contains matld (Fig. 1), the

210 introns studied here have group IIB intron secondary structures following this model. Annotated

211 sequence alignments and draft secondary structures for the remaining introns are presented in the

212 supplementary information (Figs. S5-S16 [note that no intronic sequence data were removed to

213 simplify folding]). As expected, the P. purpureum IEPs are located in the domain IV (DIV) loop,

214 which is integral for ribozyme activity. DIVa (the maturase binding site exclusive of the IEP [see

215 Fig. S23]) and DV contain conserved regions (96 $\pm 4 \%$ identity), whereas DVI is highly variable

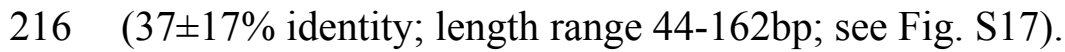

217 The bulged AC nucleotide pair illustrated within DV of Fig.1 is in agreement with the

218 model of Toor et al. (2008) and Keating et al. (2010), however the possibility exists that (as in

219 the remaining introns [Figs. S5 - S16]) the unpaired nucleotides can be shifted downstream to

220 create a CG bulge. The DVI domain contains a conserved, bulged adenosine that serves as a

221 nucleophile during lariat generation upon splicing (Peebles et al. 1986; Robart et al. 2014), 
222 however most $P$. purpreum group II intron models described here maintain an additional

223 unpaired guanine in an AG bulge. The effect this has on the splicing reaction remains unknown.

224 Structural analysis reveals a novel and unusual bipartite DIII domain configuration, because it

225 can be represented by either a canonical stem/loop structure, or as two individual stems (Figs.

226 S5-S11 [see inset DIII]), or as two individual stems only (Figs. S12-S16). The DIII domain

227 contributes an adenosine pair to a base stack that serves to reinforce DV opposite the catalytic

228 site, and stabilizes the entire structure (Robart et al. 2014). Modification of this domain in the $P$.

229 purpureum group II intron structures that have lost mobility may reflect the lack of an IEP and

230 thus need for reinforcement.

231 Group II intron RNAs self-splice via base-pairing interactions between exon-binding sites

232 (EBS1 \& EBS2) on the ribozyme and intron-binding sites (IBS1 \& IBS2) at the 5' exon region

233 (Lambowitz and Zimmerly, 2011). Despite a common origin, the P. purpureum introns that

234 encode an IEP appear to have a highly variable EBS (Fig. S18) perhaps explaining their ability to

235 spread to novel sites in these plastid genomes. Each EBS/IBS pairing is uniquely associated with

236 an intron/IEP combination, and complementarity between both is present. EBS1 and/or EBS2

237 were not identified for the matla, mat1b, and matlc introns. Interestingly, EBS1 is located at the

238 same site in the nucleotide alignment, whereas the EBS2 position is variable due to length

239 heterogeneity between introns. Understanding how variation in these binding sites affects ability

240 of group II introns to self-splice and bind target DNA is paramount for 'targetron' development

241 (Enyeart et al. 2014) and application of these mobile elements to biotechnology.

242 Finally, sequence alignment of the P. purpureum introns described here with the five

243 Rhodomonas salina introns presented in Khan and Archibald (2008) (Fig. S17) demonstrates that

244 the domain organization and secondary structure of these elements in both species are similar. 
245 We were thus able to derive amended secondary structures for the cryptophyte models proposed

246 by Maier et al. (1995) and Khan and Archibald (2008) using P. purpureum as a guide. In doing

247 so, we identified a cryptophyte domain IVa similar to that in P. purpureum that contains the IEP

248 and has modified domains DII and DIII (e.g., Fig. S19). We propose that the non-canonical

249 features described by Khan and Archibald (2008) in R. salina and H. andersenii (i.e., domain

250 insertions, ORF relocation, absence of internal splicing) can be explained by degeneration of the

251 endonuclease domain between the protein C-terminus and domain IVa. Amended structures for

252 the remaining cryptophyte introns are presented herein (Figs. S19-S23).

253

254 Red algal twintrons

255 Introns nested within other introns (or twintrons) were first reported in the Euglena gracilis

256 plastid (Copertino and Hallick, 1991). Since then, group II/III twintrons have been reported at

257 multiple sites in complete Euglenozoa plastid genomes (E. gracilis and Monomorphina

258 aenigmatica; Pombert et al. 2012) and from the plastid genomes of the cryptophytes

259 Rhodomonas salina and Hemiselmis andersenii (Maier et al. 1995; Khan et al. 2007 [however

260 see discussion, above]). Twintrons have also been described in the prokaryotes

261 Thermosynechococcus elongatus, a thermophilic cyanobacterium (Mohr et al. 2010) and in

262 Methanosarcina acetivorans, an archaebacterium (Dai and Zimmerly, 2003). Here we provide

263 the first description of twintrons in rhodophyte plastid genomes, and the first known report of an

264 inner intron (mat lf) found nested within two different outer introns (while also inserted in a third

265 gene). The plastid genomes of three $P$. purpureum strains each contain two twintrons encoding

266 matlfa and matlfb (Fig. 2A, 2B) that are bounded by different outer introns inserted in the

$267 r p o C 2$ and atpI genes, respectively. Two strains contain a copy of the inner intron/IEP inserted 
268 singly within the $a t p B$ gene as matlfc. Alignment of the outer and inner twintron regions

269 together with the other introns shows that the two different twintrons have very similar structures

270 (Fig. S1). Despite partial sequence similarity (78.2\% sequence identity in pairwise comparisons),

271 the two outer introns have similar IEP remnants. The IEPs are truncated at the same site, likely

272 due to a partial protein deletion. Approximately $130 \mathrm{nt}$ and $555 \mathrm{nt}$, respectively, remain in the 5'

273 and 3' regions of the former IEP in the external introns. Presumably, the later insertion of the

274 inner intron happened at the same binding site ( $85 \mathrm{nt}$ further downstream from the excision site).

275 Our analyses show that the closely related outer introns int.b (atpI) and int.b (rpoC2; Fig. 3) in $P$.

276 purpureum retain IEP remnants that have been truncated in the same region due to inner intron

277 insertion at the same DIV target site (Fig. S17). Of future interest is to study the splicing of these

278 red algal twintrons to confirm that excision occurs in consecutive steps as in other chloroplast

279 twintrons (Copertino et al. 1992).

281 Conclusions

282 In summary, our results support a relatively simple explanation for the origin of a complex

283 family of group II introns in the plastid genome of different P. purpureum strains (see Fig. 2A).

284 We suggest that the common ancestor of these five strains contained several IEP-encoding group

285 II introns that may trace their origin to the cyanobacterial primary plastid endosymbiont. In turn,

286 the Cryptophyta may have acquired these group II introns during the secondary endosymbiosis

287 of a red alga potentially related to a Porphyridium-like donor. These hypotheses require testing

288 with additional plastid genome data from red algae and cryptophytes. Regardless of the time or

289 mode of origin our data suggest that seeds for nuclear spliceosomal introns exist in red algae vis-

290 à-vis organelle encoded group II introns. 
It is also clear that during evolution, some mobile group II introns lose their IEP either by

292

293

294

295

296

297

298

299

300

301

302

303 304 (Lapidot et al. 2002).

complete deletion, partial degeneration (i.e., loss of the YADD motif), or by point mutations that

resulted in-frame stop codons (as in the En domain). All of these events create mobility-impaired introns that are stably inherited in descendant lineages. However, some $P$. purpureum IEPs recovered here have not undergone deleterious change and apparently retain mobility. These mobile introns are inserted in different genes in the plastid genomes, including the intron encoding the matlf IEP that created two different twintron combinations. We suggest that $P$.

purpureum is a potentially valuable eukaryote model for understanding the evolution of recently mobile group II introns. The presence of active IEPs in the P. purpureum plastid genome also makes this species a good candidate for biotechnological applications, for example via the insertion of IEP encoded foreign genes in plastid genomes (Enyeart et al. 2014). In this regard, $P$. purpureum synthesizes compounds of interest such as unsaturated fatty acids and photosynthetic pigments (Lang et al. 2011) and plastid transformation is stable, which is rare for red microalgae

\section{Acknowledgments}

307 We thank Nicolas Toro for sharing his RT domain-based IEP protein alignment. We are grateful 308 to members of the Genome Cooperative at the Rutgers School of Environmental and Biological

309 Sciences for supporting this research. The authors have no conflict of interest with respect to this 310 work.

\section{References}

312 Altschul SF, Gish W, Miller W, Myers EW, Lipman DJ. 1990. Basic local alignment search tool. 313 Journal of Molecular Biology 215: 403-410. 
314 Archibald, JM. 2009. The puzzle of plastid evolution. Current Biology 19:R81-88.

315 Bhattacharya D, Yoon HS, and Hackett JD. 2004. Photosynthetic eukaryotes unite:

316 endosymbiosis connects the dots. Bioessays 26:50-60.

317 Bhattacharya D, Price DC, Chan CX, Qiu H, Rose N, Ball S, Weber AP, Arias MC, Henrissat B,

318 Coutinho PM, Krishnan A, Zäuner S, Morath S, Hilliou F, Egizi A, Perrineau MM, Yoon

319 HS. 2013. Genome of the red alga Porphyridium purpureum. Nature Communications

$320 \quad 4: 1941$

321 Byun Y, Han K. 2009. PseudoViewer3: generating planar drawings of large-scale RNA

322 structures with pseudoknots. Bioinformatics 25:1435-1437.

323 Cech TR. 1986. The generality of self-splicing RNA: Relationship to nuclear mRNA splicing.

$324 \quad$ Cell 44:207-210.

325 Copertino DW, Hallick RB. 1991. Group II twintron: an intron within an intron in a chloroplast

326 cytochrome b-559 gene. The EMBO Journal 10:433-442.

327 Copertino DW, Shigeoka S, Hallick RB. 1992. Chloroplast group III twintron excision utilizing

328 multiple 5'- and 3'-splice sites. The EMBO Journal 11:5041-5050.

329 Dai L, Zimmerly S. 2003. ORF-less and reverse-transcriptase-encoding group II introns in

330 archaebacteria, with a pattern of homing into related group II intron ORFs. RNA 9:14-19.

331 Dai L, Toor N, Olson R, Keeping A, Zimmerly S. 2003. Database for mobile group II introns.

$332 \quad$ Nucleic Acids Research 31:424-426.

333 Doolittle WF. 2014. The trouble with (group II) introns. Proceedings of the National Academy of

$334 \quad$ Sciences of the United States of America 111:6536-6537. 
335 Enyeart PJ, Mohr G, Ellington AD, Lambowitz AM. 2014. Biotechnological applications of 336 mobile group II introns and their reverse transcriptases: gene targeting, RNA-seq, and 337 non-coding RNA analysis. Mobile DNA 5:2.

338 Jones RF, Speer HL, Kuyr W. 1963. Studies on the growth of the red alga Porphyridium cruentum. Physiologia Plantarum 16:636-643.

340 Khan H, Archibald JM. 2008. Lateral transfer of introns in the cryptophyte plastid genome. Nucleic Acids Research 36:3043-3053.

342 Khan H, Parks N, Kozera C, Curtis BA, Parsons BJ, Bowman S, Archibald J. 2007. Plastid genome sequence of the cryptophyte alga Rhodomonas salina CCMP1319: lateral transfer of putative DNA replication machinery and a test of chromist plastid phylogeny.

Lambowitz AM, Zimmerly S. 2011. Group II introns: mobile ribozymes that invade DNA. Cold Molecular Biology and Evolution 24:1832-1842. Spring Harbor Perspectives in Biology 3:a003616.

Lang I, Hodac L, Friedl T, Feussner I. 2011. Fatty acid profiles and their distribution patterns in microalgae: a comprehensive analysis of more than 2000 strains from the SAG culture collection. BMC Plant Biology 11:124.

Lapidot M, Raveh D, Sivan A, Arad SM, Shapira M. 2002. Stable chloroplast transformation of 353 Larkin MA, Blackshields G, Brown NP, Chenna R, McGettigan PA, McWilliam H, Valentin F, the unicellular red alga Porphyridium species. Plant Physiology 129:7-12. Wallace IM, Wilm A, Lopez R, Thompson JD, Gibson TJ, Higgins DG. 2007. Clustal W and Clustal X version 2.0. Bioinformatics 23:2947-2948. 
356 Maier, UG, Rensing SA, Igloi GL, Maerz M. 1995. Twintrons are not unique to the Euglena

357

358

359

360

361

362

363

364

365

366

367

368

369

370

371

372

373

374

375

376

377

378

chloroplast genome: structure and evolution of a plastome cpn60 gene from a cryptomonad. Molecular \& General Genetics 246:128-131.

Mohr G, Ghanem E, Lambowitz AM. 2010. Mechanisms used for genomic proliferation by thermophilic group II introns. PLoS Biology 8:e1000391.

Moran JV, Zimmerly S, Eskes R, Kennell JC, Lambowitz AM, Butow RA, Perlman PS. 1995. Mobile group II introns of yeast mitochondrial DNA are novel site-specific retroelements. Molecular and Cell Biology 15:2828-2838.

Pombert JF, James ER, Janouškovec J, Keeling PJ. 2012. Evidence for transitional stages in the evolution of euglenid group II introns and twintrons in the Monomorphina aenigmatica plastid genome. PLoS One 7:e53433.

Qu G, Dong X, Piazza CL, Chalamcharla VR, Lutz S, Curcio MJ, Belfort M. 2014. RNA-RNA interactions and pre-mRNA mislocalization as drivers of group II intron loss from nuclear genomes. Proceedings of the National Academy of Sciences of the United States of America 111:6612-6617.

Ragan MA, Bird CJ, Rice EL, Gutell RR, Murphy CA, Singh RK. 1994. A molecular phylogeny of the marine red algae (Rhodophyta) based on the nuclear small-subunit rRNA gene. Proceedings of the National Academy of Sciences of the United States of America 91:7276-7280.

Robart AR, Chan RT, Peters JK, Kanagalaghatta RR, Toor N. 2014. Crystal structure of a eukaryotic group II intron lariat. Nature 514:193-197

Rogozin IB, Carmel L, Csuros M, Koonin EV. 2012. Origin and evolution of spliceosomal introns. Biology Direct 7:11. 
379 Sharp PA. (1991) Five easy pieces. Science 254:663.

380 Simon DM, Kelchner SA, Zimmerly S. 2009. A broadscale phylogenetic analysis of group II

381 intron RNAs and intron-encoded reverse transcriptases. Molecular Biology and Evolution

$382 \quad 26: 2795-2808$.

383 Tajima N, Sato S, Maruyama F, Kurokawa K, Ohta H, Tabata S, Sekine K, Moriyama T, Sato N.

384 2014. Analysis of the complete plastid genome of the unicellular red alga Porphyridium 385 purpureum. Journal of Plant Research 127:389-397.

386 Tamura K, Stecher G, Peterson D, Filipski A, Kumar S. 2013. MEGA6: Molecular Evolutionary

387 Genetics Analysis version 6.0. Molecular Biology and Evolution 30:2725-2729.

388 Toro N, Martínez-Abarca F. 2014. Comprehensive phylogenetic analysis of bacterial group II

389 intron-encoded ORFs lacking the DNA endonuclease domain reveals new varieties. PLoS

$390 \quad$ One 8:e55102.

391 Toor N, Hausner G, Zimmerly S. 2001. Coevolution of group II intron RNA structures with their 392 intron-encoded reverse transcriptases. RNA 7:1142-1152.

393 Toor N, Keating KS, Taylor SD, Pyle AM. 2008. Crystal structure of a self-spliced group II

394 intron. Science 320:77-82.

395 Zuker M. 2003. Mfold web server for nucleic acid folding and hybridization prediction. Nucleic $396 \quad$ Acids Research 31:3406-3415.

397

398

399

400 
Figure 1 (on next page)

P. purpureum group IIB intron structure.

Predicted structure of the $r p o C 1$ intron containing the matld IEP. The structure is composed of six conserved domains (DI-DVI). Exon and intron binding site (EBS and IBS) and Greek letters indicate nucleotide sequences involved in long-range tertiary interactions. The IEP is located in the DIV domain 


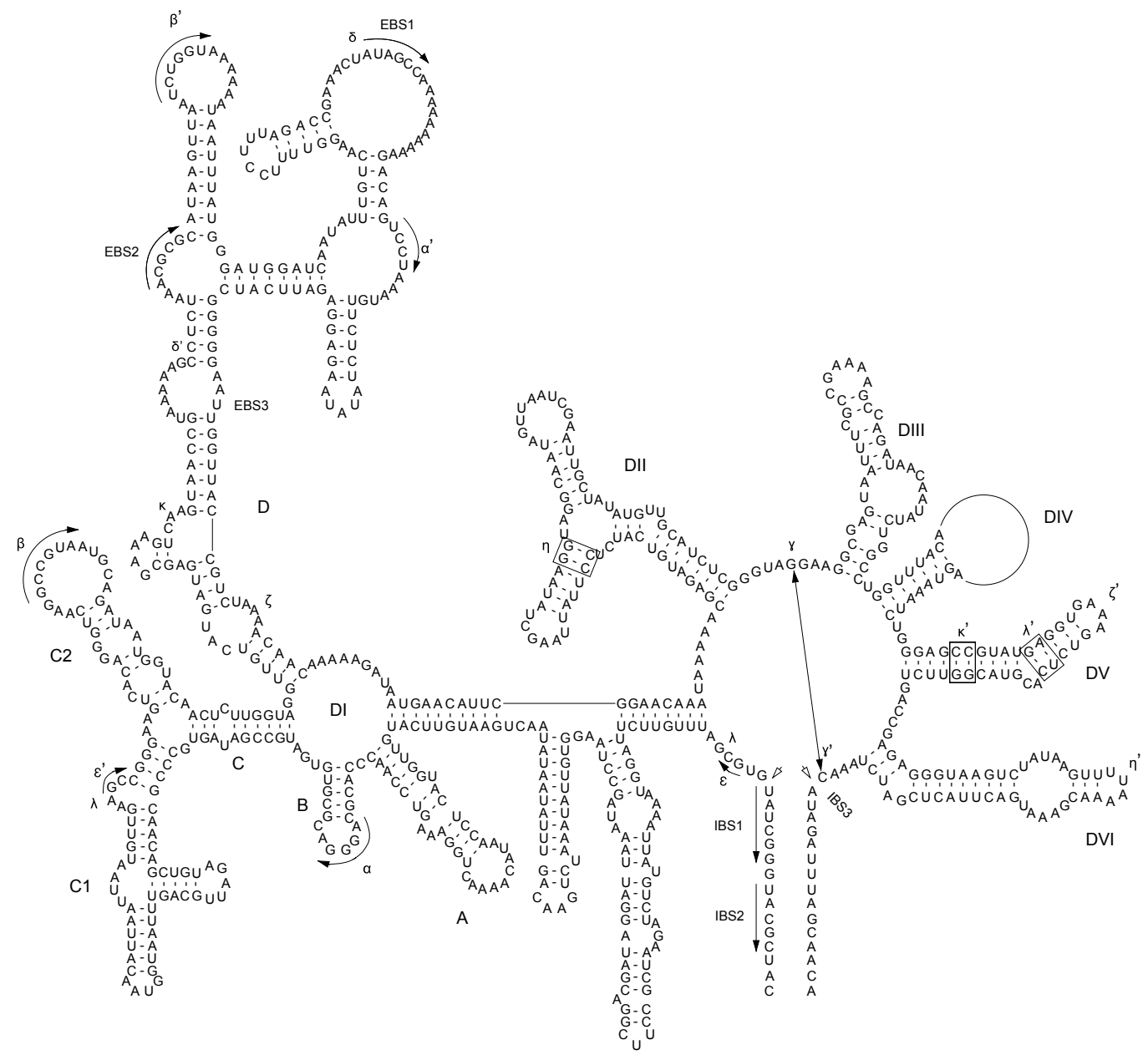




\section{Figure 2 (on next page)}

Evolution of group II introns and IEPs in Porphyridium strains.

(A) Neighbor-joining phylogenetic tree (uncorrected $p$-distance, 100 bootstrap replicates, branch supports $>70 \%$ shown) built using 332 SNPs identified in these plastid genomes. Blue arrows illustrate the distribution of group II introns containing IEPS or IEP remnants described by Tajima et al. (2014); green arrows denote group II introns containing IEPs newly described in this study, and orange arrows illustrate twintrons defined here. (B) Location of group II introns/IEPs (from Fig. 2A) in the plastid genomes (not to scale). Blue arrows illustrate the distribution of group II introns containing IEPs or IEP remnants described by Tajima et al. (2014); green arrows denote group II introns containing IEPs newly described in this study, and orange arrows illustrate twintrons defined here. 


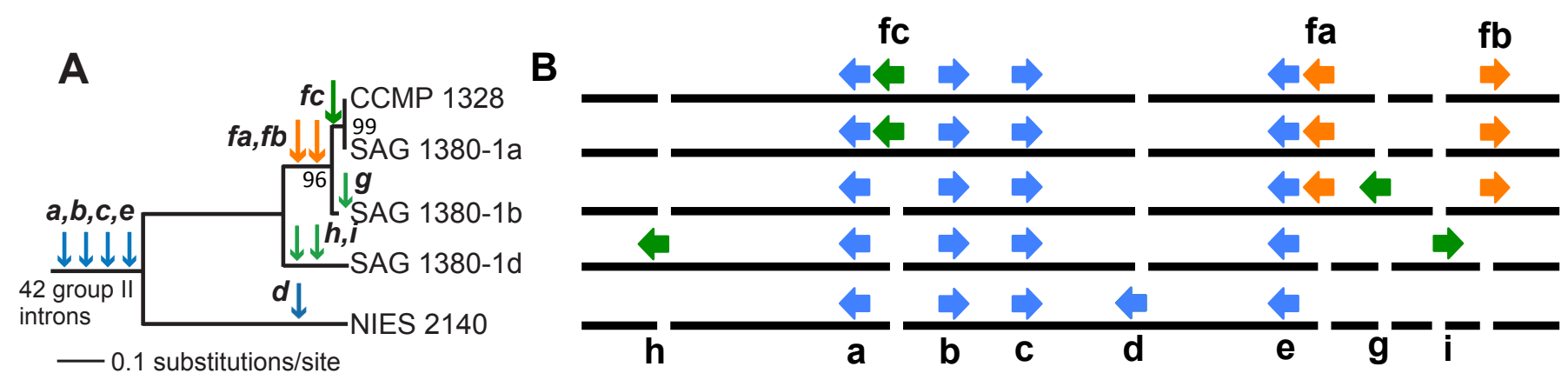

mat1a: intron in intergenic region, atpB-atpE mat1b: int.b in dnaK gene

mat1fa: inserted in maturase-lacking rpoC2 int.b (twintron)

mat1c: int.c in infC gene

mat1d: single intron in gltB gene

mat1e: int.a in rpoC2 gene mat $1 \mathrm{fb}$ : inserted in maturase-lacking atp/ int.b (twintron) mat1fc: inserted in novel intron in atpB mat1g: inserted in novel intron in rpoC1 mat1 $h$ : inserted in novel intron in ycf46 mat1i: inserted in novel intron in tsf 
Figure 3 (on next page)

Phylogeny of $P$. purpureum group II introns.

Maximum likelihood tree; only bootstrap values $>70 \%$ are shown. To avoid long-branch attraction, the IEP or IEP remnant sequences (indicated in bold) were removed from the alignment. Colored circles indicate presence (blue) or absence (red) of DNA-binding domain, Endonuclease domain and intact YADD motif, respectively. 


\section{PeerJ Reviewing Manuscript}

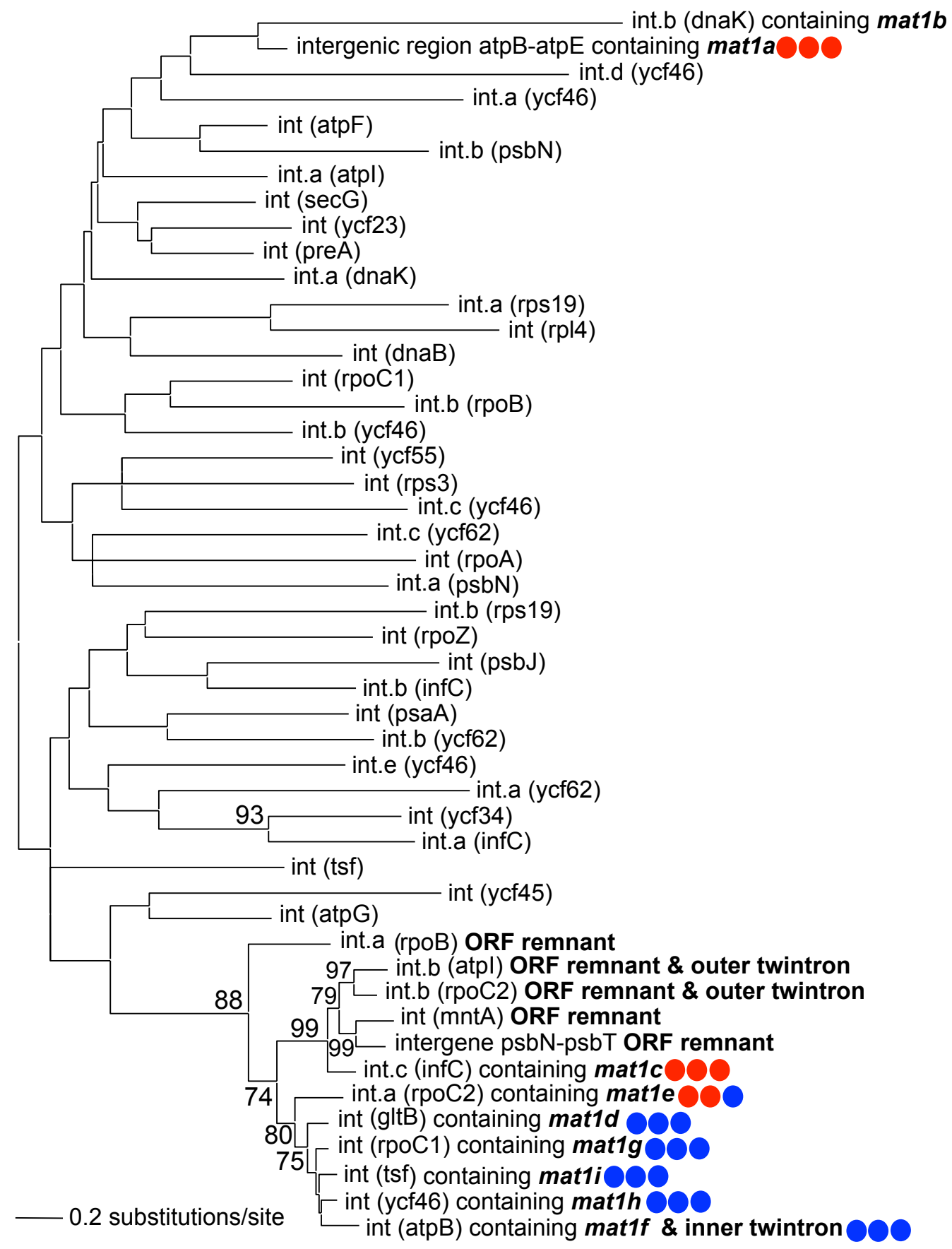


Figure 4 (on next page)

Phylogeny of CL2B group II IEPS.

The nine plastid-encoded IEP sequences from $P$. purpureum were added to selected sequences from the bacterial group II intron database, together with Cryptophyta and Euglenozoa IEPs (ML, bootstrap >70\%). The tree is rooted with proteins from the CL2A, CL1A, and CLIB groups (including the mat1b IEP). Note: the mat1f IEP represents the three nearly identical IEP sequences (matlfa, mat1fb, matlfc) described in the text. Colored circles indicate presence (blue) or absence (red) of DNA-binding domain, Endonuclease domain and intact YADD motif, respectively. 


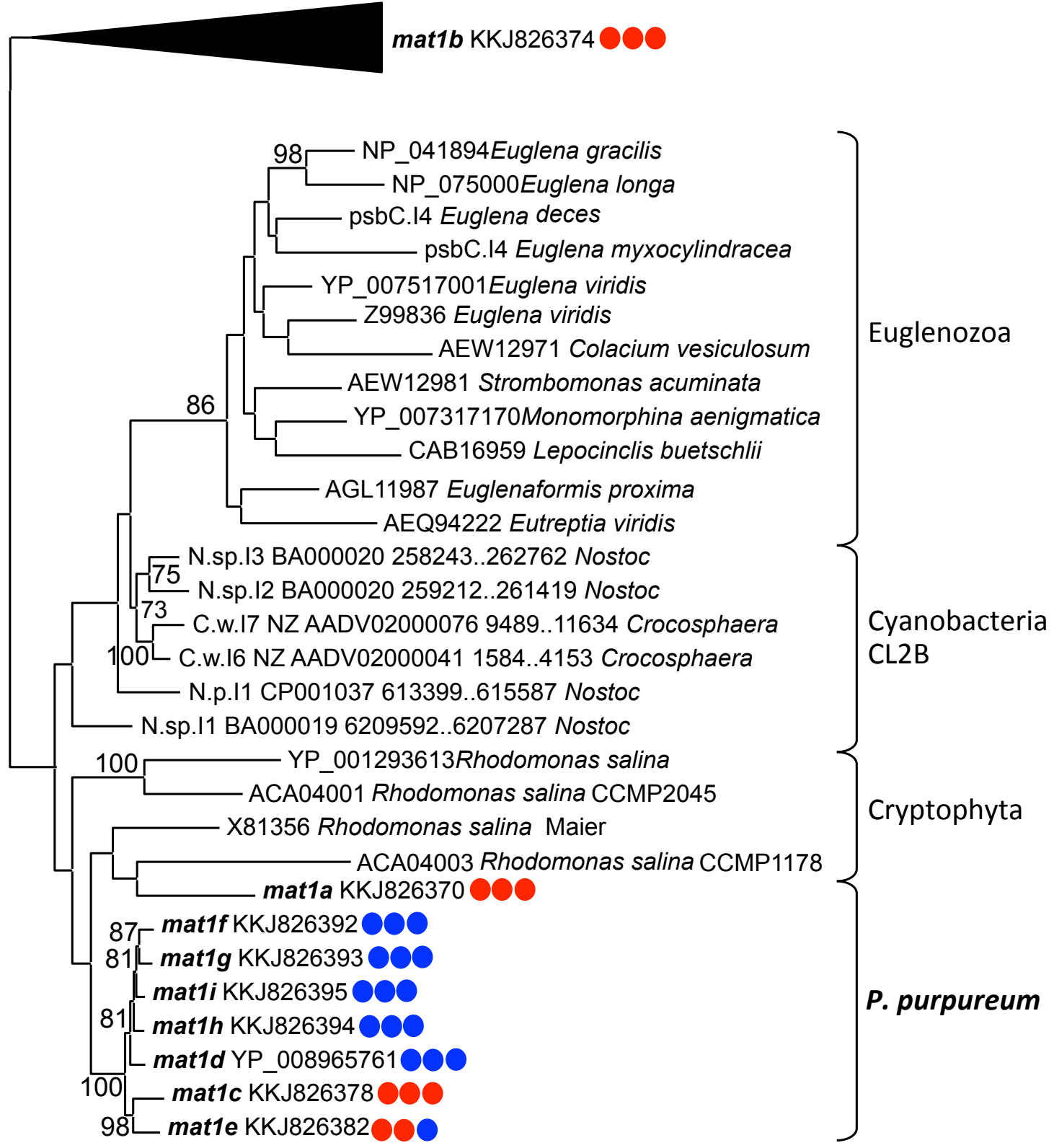

- 0.4 substitutions/site 


\section{Table $\mathbf{1}$ (on next page)}

Porphyridium purpureum plastid sequencing data generated.

Illumina sequencing data generated for each P. purpureum strain referenced in this study. 
PeerJ Reviewing Manuscript

\begin{tabular}{lrrrrrrr} 
Strain & total reads & trimmed reads & reads mapped & ref. length & \% ref covered & avg. cov. & \% ref $\geq 10 x$ cov \\
\hline SAG 1380-1a & $5,665,926$ & $5,539,699$ & 74,904 & 212,133 & 97.4 & 52.87 & 84 \\
SAG 1380-1b & $3,639,740$ & $3,639,740$ & 72,186 & 215,863 & 99.2 & 40.76 & 95 \\
SAG 1380-1d & $2,827,948$ & $3,696,004$ & 54,422 & 215,440 & 90 & 35.14 & 88 \\
CCMP 1328 & $4,524,336$ & $4,350,554$ & 83,716 & 216,010 & 99.2 & 77.41 & 96
\end{tabular}




\section{Table 2 (on next page)}

Group II introns and associated features.

IEP-containing group II introns from Tajima et al. 2014 (TEA) and IEP or IEP remnant containing group II introns described in this study are listed. Presence of reverse transcriptase (RT), maturase (MAT), endonuclease (En) and YADD motif are noted. 


\begin{tabular}{|c|c|c|c|c|c|c|c|c|}
\hline IEP & Reference & Location & IEP present? & RT & MAT & DNA & En & YADD \\
\hline mat1a & TEA & intergenic atpB-atpE & YES & YES & YES & TRUNCATED & NO & ISDQ \\
\hline mat1b & TEA & int.b dnaK & YES & YES & YES & TRUNCATED & NO & FGNK \\
\hline mat1c & TEA & int.c infC & YES & YES & YES & TRUNCATED & NO & YVDD \\
\hline mat1d & TEA & int $g / t B$ & YES & YES & YES & YES & YES & YADD \\
\hline mat1e & TEA & int.a rpoc2 & YES & YES & YES & TRUNCATED & NO & YADD \\
\hline mat1fa & This study & int.b rpoc2 & YES & YES & YES & YES & YES & YADD \\
\hline mat1fb & This study & atpl int.b & YES & YES & YES & YES & YES & YADD \\
\hline mat1fc & This study & int $\operatorname{atp} B$ & YES & YES & YES & YES & YES & YADD \\
\hline mat1g & This study & int rpoc1 & YES & YES & YES & YES & YES & YADD \\
\hline mat1h & This study & int $y c f 46$ & YES & YES & YES & YES & YES & YADD \\
\hline mat1i & This study & int tsf & YES & YES & YES & YES & YES & YADD \\
\hline no IEP & This study & intergenic $p s b B-p s b T$ & NO ('GHOST') & $\mathrm{N} / \mathrm{A}$ & N/A & N/A & $\mathrm{N} / \mathrm{A}$ & N/A \\
\hline no IEP & This study & int $m n t A$ & NO ('GHOST') & $\mathrm{N} / \mathrm{A}$ & N/A & N/A & $\mathrm{N} / \mathrm{A}$ & $\mathrm{N} / \mathrm{A}$ \\
\hline no IEP & This study & int.a $r p o B$ & NO ('GHOST') & $\mathrm{N} / \mathrm{A}$ & N/A & $\mathrm{N} / \mathrm{A}$ & $\mathrm{N} / \mathrm{A}$ & $\mathrm{N} / \mathrm{A}$ \\
\hline
\end{tabular}

